\title{
Momonisia phreatica n. gen., n. sp. (Momoniidae, Hydrachnellae) des eaux souterraines de Bulgarie
}

par

\author{
A. PETROVA*
}

\section{SUMMARY}

\begin{abstract}
Momonisia phreatica n.gen., n.spec. (Momoniidae, Hydrachnellae) of Subterranean Waters of Bulgaria

Recent borings in the Veleka River drainage area (South Eastern Bulgaria) have shown a phreatic fauna with a new representative of Hydracarian of the family Momoniidae which constitue the type of a new genus: Momonisia phreatica n. gen. $\mathrm{n}$. sp. The description is here given by the author. The most closely related Momoniid appears to be Momonia karelica Sokolov from Russian Carelia.
\end{abstract}

Récemment les sondages pour la récolte de la faune interstitielle effectués dans le bassin de la rivière Véléka m'ont permis de trouver un nouveau représentant de la famille Momoniidae. Son examen détaillé m'a convaincu qu'il constituait le type d'un genre nouveau - Momonisia phreatica n. gen. n. sp., dont la description est donnée ci-après.

La famille des Momoniidae comprend 4 genres: Momonia, Momoniella, Stygomomonia et Momonides. Le nouveau genre semble être le plus rapproché du genre Momonia, spécialement de l'espèce Momonia karelica (Sokolov, 1926), en ce qui concerne la structure et la disposition des épimères, la structure de l'organe génital et la peau. D'autre part la structure plus particulière de la patte $I$, la forme des épimères, les pattes II, III et IV dépourvues de soies natatoires etc. m'ont persuadée que le statut générique de Momonisia est justifié et nécessaire.

Je remercie sincèrement $\mathrm{Mr}$ le Professeur Bader de m'avoir confirmé le nouveau taxon et procuré aimablement la photocopie du travail de Cook (1966).

\section{Genre Momonisia nov.}

Diagnose: Corps ovale de structure épineuse, absence de cuiraces dorsale et ventrale. Sur le bord latéral du corps 8 paires de papilles sensorielles, à la partie dorsale 4 paires de plaquettes chitineuses et 4 paires de glandules. Le $2^{\text {me }}$ article du palpe est égal au $4^{\mathrm{me}}$, les deux épines fortes sur le $4^{\mathrm{me}}$ article - de petite taille. Les épimères

\footnotetext{
*Institut zoologique et Musée, 1 Boul. Rouski, SOFIA - Bulgarie
} 
ne dépassent pas le bord frontal du corps. Les bords internes des épimères ne contactent pas la ligne médiane. L'organe génital - situé à une petite distance du bord postérieur du Ep. IV, $3+3$ disques génitaux, manque de plaques postgénitales. Pattes I avec $5^{\mathrm{e}}$ article en forme de massue, le $6^{\mathrm{e}}-$ profondément entaillé, très allongé, avec une seule griffe puissante et mobile qui forme une pince avec l'article.

\section{Momonisia phreatica n. gen. n. sp.}

Matériel: holotype femelle, paratypes - 5 femelles, 1 mâle, le 28.11.1973, sondages exécutés à l'aide d'une modification de l'appareil de Bou et Rouch, dans les alluvions de la rivière Mladejka, affluent de la rivière Véléka, à un mètre de distance de la rive, aux environs du village Mladejko (Bulgarie du Sud-Est); 19. VII. 1973, 5 femelles, 1 mâle - même localité; le 3.XI.1973 - 1 femelle - même localité.

Les types sont déposés dans la collection d'Acariens de l'Institut de Zoologie de l'Académie bulgare des Sciences à Sofia.

Description: Corps de forme ovale. Couleur - pâle-rose-jaunâtre. La longueur dorsale sans capitulum - $718 \mu$, la largeur de $613 \mu$. (Les dimensions de quelques femelles mesurées varient entre 655-718 $\mu$ de long sur 549-613 $\mu$ de large). Les yeux sont petits, noirs. La cuirace est absente. La peau molle est couverte de petites épines. Sur le bord latéral de chaque côté de la ligne médiane se trouvent 8 papilles sensorielles. Les papilles frontales sont grandes et dépourvues de poils. Sur la partie dorsale on trouve 4 paires de plaquettes chitineuses, dont la III $^{\mathrm{e}}$ est située plus à l'extérieur de la ligne médiane, comme d'ailleurs 4 paires de glandules accompagnées d'un poil. L'organe maxillaire est à rostre court. La mandibule est longue de $153 \mu$. Elle ressemble beaucoup à la mandibule de Momonia karelica (Lundblad, 1962, p.215). Les caractères généraux du palpe sont identiques à ceux des autres Momoniidés. La longueur dorsale des articles en $\mu$ est: $\mathrm{P}_{1}-17 \mu, \mathrm{P}_{2}-58$ $\mu, \mathrm{P}_{\square}-33 \mu, \mathrm{P}_{4}-58 \mu, \mathrm{P}_{5}-50 \mu$. Le $1^{\mathrm{e}}$ article porte une soie dorsale à l'extrémité distale. Le $2^{\mathrm{e}}$ article est égal au $4^{\mathrm{e}}$. II est muni de cinq soies fortes sur son bord dorsal, dont deux sont insérées près de l'extrémité proximale, deux à l'extrémité distale et une de position médiane. Pareille disposition des soies se trouve chez Momonia falcipalpis Halb. (Motas, 1928, p.212). Le $3^{\mathrm{e}}$ article porte quatre soies dorsales - deux d'une position médiane et deux de position distale. Ces dernières ne dépassent pas le bord distal du $4^{\mathrm{e}}$ article (chez $M$. karelica les soies distales du $3^{\mathrm{e}}$ article sont plus longues que le $4^{\mathrm{e}}$ article (Lundblad, 1962, p.215)). Le $4^{\mathrm{e}}$ article est caractérisé par un petit renflement à la partie ventrale qui porte les deux épines fortes propres à la famille des Momoniidés. Sur le bord dorsal il est pourvu de deux soies, une médiane et une distale. Le $5^{\mathrm{e}}$ article se termine en crochet et porte aussi deux petites épines à la partie ventrale et une plus mince dorsale, insérée à l'extrémité distale.

La région épimérale couvre la moitié de la partie ventrale du corps. Les épimères ne dépassent pas le bord frontal du corps. Leur bords internes ne viennent pas en contact sur la lige médiane. L'espace entre Ep.II et Ep.III est perçée d'une ouverture glandulaire. Sur le champ postépiméral se trouvent 3 paires de glandules, dont les deux antérieures sont accompagnées d'un poil. 


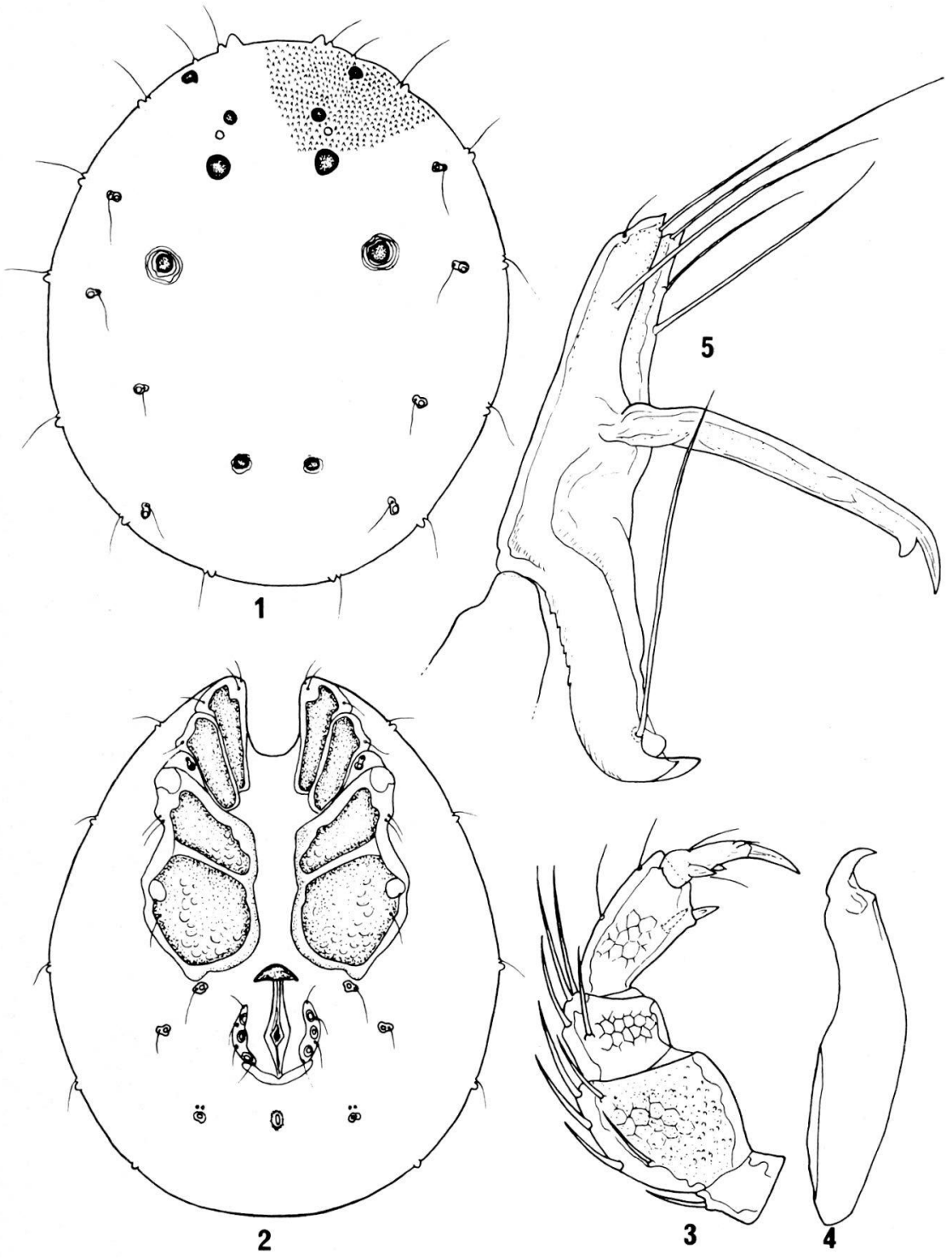

PLANCHE I - Momonisia phreatica n. gen. n. sp.?

1 - face dorsale

2 - face ventrale

3 - palpe

4 - mandibule

5 - article $6^{\mathrm{e}}$ de patte I 


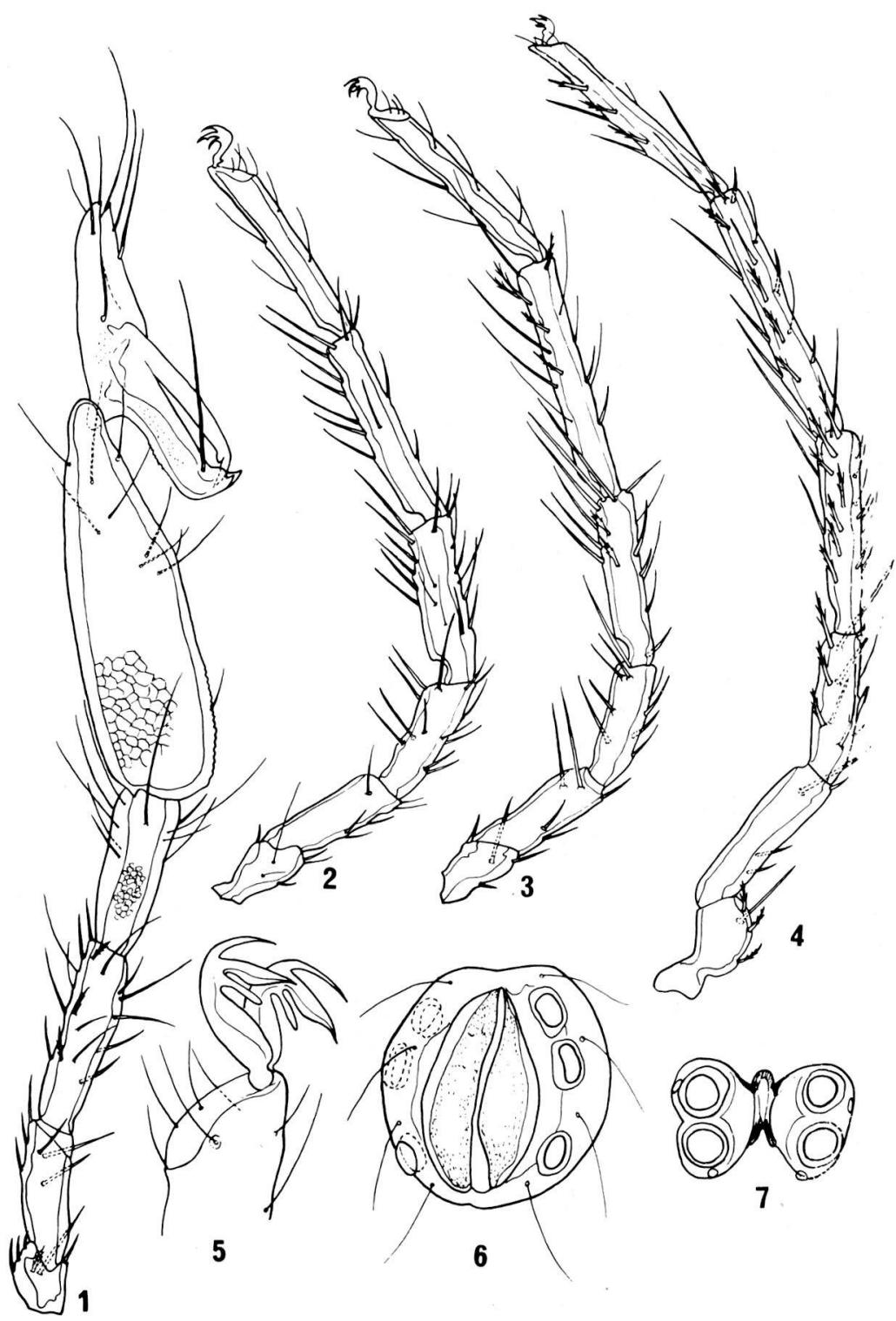

PLANCHE II - Momonisia phreatica n. gen. n. sp. (I-5 $\$ ; 6-\delta ; 7-$ Ny)

1 - patte I

2 - patte II

3 - patte III

4 - patte IV

5 - les griffes de la patte III

6 - l'organe génital du mâle

7 - l'organe génital de la nymphe 
L'organe génital est situé à une petite distance du bord postérieur de Ep.IV. II mesure $149 \mu$ de long sur $124 \mu$ de large. La fente génitale est grande, les plaques génitales de forme allongée portent chacune 3 disques génitaux et 4 petits poils.

Le pore excréteur se trouve entre les deux glandules postérieures.

Les pattes antérieures sont longues, le $5^{\mathrm{e}}$ article en forme de massue, le $6^{\mathrm{e}}$ présente à son extrémité une entaille profonde au début de laquelle est insérée la griffe. Cette dernière est très allongée et se termine par un crochet et une dent massive. La griffe est mobile et forme une sorte de pince avec l'entaille proximale de l'article. Le dernier article en raison de son articulation particulière avec le $5^{\mathrm{e}}$ est très mobile aussi autour de son axe. La structure bizarre de $6^{\mathrm{e}}$ article de la première patte, rend le nouveau genre très différent des autres genres de la famille.

Les trois dernières pattes portent des soies fortes, ainsi que des soies fines en nombre réduit. C'est seulement sur les pattes III et IV que se trouvent des soies denticulées. Les pattes sont privées des soies natatoires à cause de la vie souterraine interstitielle. Les griffes des II, III et IV pattes sont à trois dents dont la médiane est la plus développée.

La longueur des pattes en $\mu$ est comme suit:

$\begin{array}{lrrrrrr} & \mathrm{B}_{1} & \mathrm{~B}_{2} & \mathrm{~B}_{3} & \mathrm{~B}_{4} & \mathrm{~B}_{5} & \mathrm{~B}_{6} \\ \text { I } & 41 & 91 & 141 & 132 & 298 & 149 \\ \text { II } & 54 & 83 & 99 & 124 & 160 & 166 \\ \text { III } & 66 & 99 & 99 & 133 & 182 & 174 \\ \text { IV } & 83 & 107 & 124 & 174 & 199 & 207\end{array}$

Mâle: Sauf l'organe génital et la taille plus petite, la structure du corps n'accuse pas de différences avec la femelle. La longueur dorsale sans l'organe maxillaire est de $605 \mu$, la largeur de $563 \mu$. La longueur dorsale des articles du palpe est: $P_{1}-16$ $\mu, \mathrm{P}_{2}-50 \mu, \mathrm{P}_{3}-33 \mu, \mathrm{P}_{4}-50 \mu, \mathrm{P}_{5}-46 \mu$. L'organe génital est de forme ovale, long de $91 \mu$ et large de $90 \mu$. Il porte 3 paires de disques génitaux et 4 paires de longs poils.

La nymphe ressemble à la forme adulte par l'aspect général du corps, la conformation des palpes et des pattes préhensiles, par la forme de la région épimérale. L'organe génital provisoire est pourvu de deux plaques génitales réunies, portant deux disques chacune.

\section{RESUME}

De récents sondages effectués dans le bassin de la rivière Veleka (Sud-Est de la Bulgarie) ont mis en évidence dans la faune interstitielle un nouveau représentant d'Hydracariens de la famille des Momoniidae, qui constitue le type d'un genre nouveau: Momonisia phreatica n. gen. n. sp. dont l'auteur donne ici la description. Le Momoniidé le plus proche semble être Momonia karelica Sokolov de la Carélie russe. 


\section{BIBLIOGRAPHIE}

ANGELIER, E., 1954 - Contribution à l'étude de la faune d'eau douce de la Corse. Acariens psammiques (Hydrachnellae et Porohalacaridae). Vie et Milieu, 4 (2):525-528.

COOK, D. R., 1966 - Water Mites of Liberia. Mem. Amer. Ent. Inst., n. 6:229-230.

COOK, D. R., 1968 - Water Mites of the Genus Stygomonia in North America. Proc. Ent. Soc. Wash., vol. 70, Nr 3:210-224.

HALBERT, J., 1906 - Notes on Irish Hydrachnida; with description of a new genus and two new species. Ann. Mag. Natur. Hist. London (s.7), 18:4-18.

HABEEB, H., 1961 - Walter Vincent Powers, Noble Fellow, Leaflets of Acadian Biology, Nr 22:5-6, Holywood, California.

IMAMURA, T., 1957 - Subterranean Water-Mites of the Middle and Southern Japan. Arch. f. Hydrobiol., 53,3: 376-378.

LUNDBLAD, O., 1941 - Neue Wassermilben. Vorläufige Mitteilung, Ent. Tidskr., 62,1-2:97.

LUNDBLAD, O., 1962 - Die Hydracarinen Schwedens. II. Arkiv för Zoologi, Bd. 14 (1-6):215-216. Stockholm.

LUNDBLAD, O., 1971 - Weitere Beiträge zur Kenntnis der Fliesswassermilben Javas. Arkiv för Zoologi, S. 2, Bd. 23, nr 5:351-352.

MOTAS, C., 1928 - Contribution à la connaissance des Hydracariens français particulièrement du Sud-Est de la France. Trav. Hydrobiol. Piscic. Univ. Grenoble., 20:211-224.

MOTAS, C. et J. TANASACHI, 1964 - Acariens phréaticoles de Transylvanie. Notat. Biol., vol. IV, nr 1-3:42-51, Bucarest.

MOTAS, C., TANASACHI J. et N. BOTNARIUC, 1948 - Sur quelques Hydracariens recueillis en Yougoslavie dans le bassin de la Bosna. Bull. Ecole Polytechn. Jassy, T. 3 (2):14-19.

SZALAY, L., 1943 - Eine neue subterran lebende Wassermilbe (Hydrachnellae, Acari) aus Ungarn. Fragm. faun. Hungar. 6 (2):58-63.

SOKOLOV, I., 1926 - Eine neue Hydracarinen-Gattung aus Russisch-Karelien, Kondia nov. gen. Zool. Anz., Bd. 66, Nr 1/4:32-40.

VIETS, K., 1929 - Weitere neue Hydracarinen-Gattungen von A. THIENEMANN's (15.7) Sunda-Expedition. Zool. Anz. Bd. 83, Nr 9/10:237.

WALTER, C., 1947 - Neue Acari (Hydrachnellae, Porohalacaridae, Trombidiidae) aus subterranen Gewässern der Schweiz und Rumäniens. Verhandl. Naturforsch. Gesellsch. Basel, Bd. LVIII:225-226. 